\title{
NET-Mediated Pathogenesis of COVID-19: The Role of NETs in Hepatic Manifestations
}

\author{
Wael Alkattan ${ }^{1}$ Ahmed Yaqinuddin ${ }^{1}$ Areez Shafqat ${ }^{1}{ }^{10}$ Junaid Kashir ${ }^{1,2}$ \\ ${ }^{1}$ College of Medicine, Alfaisal University, Riyadh, Kingdom of Saudi \\ Arabia \\ 2 Department of Comparative Medicine, King Faisal Specialist Hospital \\ and Research Center, Riyadh, Kingdom of Saudi Arabia \\ Address for correspondence Ahmed Yaqinuddin, MBBS, PhD, College \\ of Medicine, Alfaisal University, Riyadh, Kingdom of Saudi Arabia \\ (e-mail: ayaqinuddin@alfaisal.edu). \\ J Health Allied Sci ${ }^{\mathrm{NU}}$ 2022;12:235-242.
}

Abstract


Keywords
- COVID-19
- SARS-CoV2
- NETs
- liver disease
- ACE2 receptor
- multi-organ failure

Some coronavirus disease-2019 (COVID-19) patients exhibit multi-organ failure, which often includes the liver. Indeed, liver disease appears to be an emerging feature of COVID-19 infections. However, the exact mechanism behind this remains unknown. Neutrophil extracellular traps (NETs) have increasingly been attributed as major contributors to various liver pathologies, including sepsis, ischemic-reperfusion (I/R) injury, and portal hypertension in the setting of chronic liver disease. Although vital in normal immunity, excessive NET formation can drive inflammation, particularly of the endothelium. Collectively, we propose that NETs observed to be elevated in severe COVID-19 infection play principal roles in liver injury in addition to acute lung injury. Herein, we discuss the potential mechanisms underlying COVID-induced liver injury including cytopathic effects from direct liver infection, systemic inflammatory response syndrome, and hypoxic injury, encompassing $I / R$ injury and coagulopathy. Further research is required to further elucidate the role of NETs in COVID. This holds potential therapeutic significance, as inhibition of NETosis could alleviate the symptoms of acute respiratory distress syndrome and liver injury, as well as other organs.

\section{Introduction}

The outbreak of severe acute respiratory syndrome coronavirus 2 (SARS-CoV-2)-mediated coronavirus disease2019 (COVID-19) has severely decimated the global health community, with data suggesting a weakened immune system in individuals presenting with increased severity of disease. ${ }^{1-4}$ Although asymptomatic in most cases, some critically ill COVID-19 patients exhibit rapid deterioration characterized by life-threatening acute respiratory distress syndrome, neutrophilia, and a cytokine storm resulting in systemic inflammatory response syndrome (SIRS), sepsis and coagulopathy, and multi-organ failure. However, the exact mechanism behind this remains unknown.

Excluding well-known respiratory symptoms of SARS-CoV-2 infections, many patients reportedly develop gastrointestinal symptoms, including diarrhea, nausea, vomiting, and abdominal pain. In some cases, such symptoms manifested earlier than the classic fever and pulmonary manifestations of COVID-19. ${ }^{5}$ Furthermore, numerous reports exist reporting hepatobiliary involvement in COVID-19 patients, as evidenced by abnormal liver function tests (LFTs), with their degree of elevation correlating with disease severity. Indeed, liver injury-manifesting as elevated LFTs with mild/moderate elevations in aspartate aminotransferase (AST) and alanine aminotransferase (ALT)-in published online

January 18, 2022
DOI https://doi.org/ 10.1055/s-0041-1741418. ISSN 2582-4287.

\footnotetext{
(c) 2022. Nitte (Deemed to be University). All rights reserved. This is an open access article published by Thieme under the terms of the Creative Commons Attribution-NonDerivative-NonCommercial-License, permitting copying and reproduction so long as the original work is given appropriate credit. Contents may not be used for commercial purposes, or adapted, remixed, transformed or built upon. (https://creativecommons.org/ licenses/by-nc-nd/4.0/) Thieme Medical and Scientific Publishers Pvt. Ltd., A-12, 2nd Floor, Sector 2, Noida-201301 UP, India
} 
COVID-19 patients is reported to range from 14.8 to $53 \% .^{6-8}$ These findings may be accompanied by slight elevations in total bilirubin and, in severe case, hypoalbuminemia. ${ }^{9}$ With disease progression from mild to severe, LFTs correspondingly increase; AST and ALT elevations are reported to be present in approximately $56 \%$ of severely affected patients. ${ }^{7}$ In short, varying degrees of liver pathology appear to be a salient feature of COVID-19 patients suffering from mild/moderate and severe infections.

Neutrophil extracellular traps (NETs)-released by neutrophils-have increasingly been attributed as major contributors to various liver pathologies, regardless of etiology, including sepsis, ischemic-reperfusion (I/R) injury, and portal hypertension. ${ }^{10}$ Historically, neutrophils were confirmed to be the first immune effector cells recruited to sites of inflammation, where they mediate host defense through degranulation (antimicrobial release) and phagocytosis of offending microbes. In 2004, however, a novel third function was identified: NETs, ${ }^{11}$ which are fibrous, DNA-based, weblike structures released by activated neutrophils by a unique form of programmed cell death termed as NETosis. NETs play vital roles in immunity by trapping and neutralizing microbes, preventing their dissemination. However, dysregulated NET production has been implicated as central to many immune-related diseases. ${ }^{12}$ Indeed, NET secretion can damage normal cells in close enough proximity through various cytotoxic elements and, consequently, propagate proinflammatory responses. $^{12}$

NET secretion is primarily induced by proinflammatory cytokines such as IL-1 $\beta$ released from macrophages secondary to inflammasome activation. Intriguingly, high levels of inflammasomes and its induced cytokines (i.e., IL-1 $\beta$ and IL18) have been observed in COVID-19 patients, possibly implicating inflammasome activation as central to the SIRS, sepsis, and coagulopathy observed in severe COVID-19. ${ }^{4}$ Considering the links between inflammasomes and NETs and the fact that neutrophilia is a cardinal feature of severe COVID-19 infections, ${ }^{13-15}$ we propose that excessive NET production by activated neutrophils participates in the pathogenesis of COVID-19, paying special attention to liver pathology. Recently, the role of NETs in COVID-19 lung injury has been confirmed by histopathological examinations. ${ }^{16,17}$ Numerous clinical trials are accordingly ongoing to evaluate the efficacy of NET-inhibiting or NET-lysing drugs in the treatment of COVID-19 patients. However, the pathomechanisms underlying systemic symptoms in severe patients still remain to be proven, which is the premise of our postulation. Future research aiming to substantiate NETs as important mediators of multi-organ pathology in severe COVID-19 could rationalize treatments for systemic COVID-19 symptoms.

\section{Neutrophil Extracellular Traps: Structure and Function} Neutrophils are the most abundant circulating immune cells and characterize acute inflammation. Neutrophils contribute to host defense primarily via phagocytosis, generation of ROS, and degranulation. However, neutrophils have been implicated in the pathophysiology in a remarkable spectrum of diseases, including cardiovascular, inflammatory, autoim- mune, metabolic, infectious, and septic conditions, through the production of NETs. ${ }^{18}$ NETs are extracellular structures produced by a programmed form of neutrophil death termed as NETosis, resulting in the extrusion of neutrophil DNA and histones, which form a fibrous structure entrapping neutrophil granule proteins such as neutrophil elastase (NE) and neutrophil cytosolic proteins such as myeloid-related protein 14 (MRP14). ${ }^{10,19}$ NETs play key roles in immune defense: histones, NE, and MRP14 exhibit bactericidal and antimicrobial properties; additionally, due to their fibrous structure, NETs occupy large amounts of space, they sequester bacteria in areas where the concentration of its antimicrobial components is high while simultaneously preventing microbial dissemination. ${ }^{12,20-22}$

Although vital in normal immunity, excessive NET formation can drive inflammation via damage-associated molecular patterns (DAMPs) and by host cell injury, particularly of the endothelium, through histones. ${ }^{23}$ Additionally, the fibrous structure of NETs constitutes a platform to which red blood cells (RBCs), platelets, fibrinogen, and fibronectin can bind, precipitating thrombus formation. ${ }^{24,25}$ Accordingly, NETs are well-established contributors to various disease processes, including vascular diseases such as atherosclerosis and hypercoagulability, metabolic diseases such as diabetes, autoimmune diseases such as systemic lupus erythematosus and rheumatoid arthritis, and, the focus of this manuscript, systemic inflammation in severe COVID-19. ${ }^{18,26}$

\section{COVID-19 and Liver Disease}

Numerous studies have assessed how the liver is affected during COVID-19, with abnormal LFTs mainly being characterized by mild elevations in serum AST/ALT. Two to eleven percent of patients with COVID-19 have pre-existing liver diseases, and $14-53 \%$ of cases feature elevated AST levels, with its magnitude of elevated correlating with disease severity. ${ }^{7}$ Indeed, a study reported AST elevations in $62 \%$ of ICU-admitted patients, compared with $25 \%$ in patients who did not require ICU admission. ${ }^{27}$ Accompanying abnormalities in serum bilirubin and, in severe cases, hypoalbuminemia, are also observed. Severe abnormalities in liver enzymes are also associated with a higher mortality. ${ }^{7,9,19,28}$ Histopathological findings of liver autopsy specimens in patients who succumbed to COVID-19 demonstrated macrovesicular steatosis with mild lobular and portal inflammation. In some cases, vascular pathologies in the form of sinusoidal microthrombi were also observed. ${ }^{8,17,29}$

Collectively, these results implicate COVID-19 liver disease as being more prevalent in severe cases compared with mild cases. Since early on during the pandemic, concerns have been documented that patients with chronic liver disease may be increasingly susceptible to severe COVID19 infections. These postulations are informed by several factors: (1) overlapping comorbidities associated with severe COVID-19 and liver diseases, including age, hypertension, and diabetes, and (2) the fact that chronic liver disease induces coagulopathy, a major risk factor for the development of severe COVID. ${ }^{30}$ Lastly, the presence and degree of 
liver injury in COVID-19 has an important bearing on treatment. Mild COVID-19 liver injury is self-limiting and typically resolves without any specific treatment; in this case, therapy aims to actively treat primary disease. In contrast, acute liver injury in severe COVID cases-considered to be due to cytokine storms and circulatory collapse-requires liver protective drugs, ${ }^{7}$ with simultaneous respiratory and circulatory support. ${ }^{31}$

The pattern and frequencies of LFT abnormalities are similar regardless of the presence of underlying chronic liver disease. ${ }^{30}$ Furthermore, the mechanism behind SARS-CoV-2induced liver disease remains uncertain but is likely multifactorial. In this article, we discuss (1) cytopathic effects from direct liver infection, (2) SIRS, and (3) in severe cases, hypoxic injury encompassing I/R injury and coagulopathy.

\section{Direct Infection}

As mentioned prior, LFT abnormalities mainly feature AST and ALT abnormalities. Intriguingly, AST and ALT elevations in COVID-19 patients do not correlate with inflammatory markers such as CRP or markers of rhabdomyolysis, hinting at the direct infection of the liver as being causal. ${ }^{30}$ The angiotensin-converting enzyme 2 (ACE2) receptor has been established as the portal of entry of SARS-CoV and SARSCoV-2 into cells. Following attachment to ACE2 to the spike (S) protein, cleavage of the $S$ protein by the transmembrane serine protease 2 allows internalization of the virus by endocytosis. $^{32}$ The tropism of SARS-CoV-2 for the liver stems from the expression of ACE2 on hepatocytes and, in particularly high levels comparable to alveolar cells of the lung, on cholangiocytes (cuboid epithelial cells of the bile duct). ${ }^{33,34}$ Furthermore, the isolation of SARS-CoV-2 RNA from stool samples of infected COVID-19 patients raises concerns about fecal-oral transmission. ${ }^{19}$

However, direct viral infection seems unlikely when correlated with the histopathologic evidence, since rather than the expected intracellular viral inclusions with concomitant lymphocytic infiltrates manifesting as piecemeal necrosis characteristic of viral hepatitis, ${ }^{35}$ histopathology reports demonstrate microvesicular and macrovesicular steatosis. These observations were made in autopsies with SARS-CoV-2 as the only risk factor, distinguishing these lesions from hepatic steatosis due to pre-existing nonalcoholic fatty liver disease (NAFLD), which is regarded as an independent risk factor for poor COVID-19 prognosis. ${ }^{36,37}$ Direct SARS-CoV-2 cytopathic effect-induced mitochondrial dysfunction has been implicated as potentially causing hepatic steatosis, a mechanism also seen in NAFLD; these findings have rationalized postulations that SARS-CoV-2 worsens pre-existing NAFLD-induced steatosis. ${ }^{38}$ Alternatively, these characteristic histopathologic patterns of liver injury may also be due to pre-existing obesity and diabetes mellitus. $^{17}$

These observations may also perhaps be due to the induction of endoplasmic reticulum (ER) stress upon the infection of the hepatocytes. This results in de novo lipogenesis. ${ }^{38}$ In the setting of SARS-2-CoV, as well as the other coronaviruses, ER stress markers glucose-regulated protein
79 (GRP78) and GRP94 are elevated. ${ }^{38,39}$ Lipogenesis may aid in viral replication and exocytosis from the cell. To this end, enhanced de novo lipogenesis has been suggested to provide the virus with the vesicular systems necessary for viral replication and exocytosis. ${ }^{38}$ Other than ER stress, lipogenesis is also induced by the mammalian target of rapamycin (mTOR) pathway. Briefly, mTOR functions as the principal intracellular nutrient sensor. When nutrient levels are high, as suggested by the presence of hormones, growth factors, and glucose, mTOR activates protein synthesis and lipogenesis. ${ }^{40,41}$ The mechanisms pertaining to mTORinduced lipogenesis involve the activation of SREBP, a membrane-bound transcription factor, which binds specific response elements to upregulate the expression of genes pertaining to cholesterol and fatty acid synthesis. ${ }^{40,42}$ In the context of COVID-19, direct infection of hepatocytes and elevated IL-6-due to the systemic cytokine storm seen in severe cases-could activate mTOR. Similar observations are seen in SARS-CoV-1 and MERS-CoV. mTOR, in turn, inhibits autophagy to prevent viral degradation in autophagolysosomes and enhances lipogenesis and protein synthesis. ${ }^{43,44}$ The elevated protein synthesis induced by mTOR involves the activation of cap-dependent translation machinery, which is essential to coronavirus replication, which hijacks this machinery. ${ }^{45}$ The de novo lipogenesis, as mentioned above, enhances viral replication and exocytosis. Accordingly, pre-existing mTOR hyperactivity-such as that seen in obese patients and diabetics-may, at least in part, explain the increased risk of severe COVID infections in these patients. ${ }^{23,24}$

However, since ACE2 is barely expressed on hepatocytes, further studies investigating SARS-CoV-2 directly infecting hepatic cells are required. Since cholangiocytic ACE2 expression is 20 times higher than hepatocytes and evidence of cholangiocyte proliferation has been observed in the setting of COVID, suggestions have been made of a compensatory proliferation of liver cells derived from the bile duct epithelium leading to an increased ACE2 expression in the liver. ${ }^{19}$ High circulating levels of IL-6, a strong cholangiocellular mitogenic factor, may contribute to the proliferative response. $^{38}$

\section{The Cytokine Storm and SIRS}

The majority of COVID-19 patients display little to no symptoms, while others suddenly deteriorate and develop severe fever and pneumonia, culminating in acute severe respiratory distress syndrome and ultimately death. Increasing evidence indicates that this presentation is probably secondary to a dysregulation of the innate immune response, resulting in a cytokine storm, systemic inflammatory response syndrome, and multi-organ failure, including the liver. Indeed, levels of numerous pro-inflammatory cytokines, such as IL$1 \beta$, IL-2, IL-6, IL-10, IL-17, TNF, and monocyte chemoattractant protein 1 are found to be elevated in patients. ${ }^{46}$

As previously intimated, the inflammasome may play a significant role in the immunopathogenesis of COVID-19, suggested by elevated serum levels of the inflammasomerelated cytokines IL-1 $\beta$ and IL-18. Furthermore, the presence 
of inflammasome-related cytokines IL-1 $\beta$ and IL-18 in COVID-19 patients correlated with disease severity. ${ }^{47,48}$ Inflammasome activation, regardless of the initiating stimulus, occurs in two steps: a priming stage, with an additional signal being required for full activation. The priming stage is constituted by the activation of pattern recognition receptors by DAMPs or pathogen-associated molecular patterns (PAMPs), which induce the activation of NF-KB promoting the gene expression of pro-IL-1 $\beta$ and pro-IL-18 and NOD-like receptor family pyrin domain-containing 3 (NLRP3). ${ }^{49-51}$

The second activation signal could be provided by several extracellular stimuli, including membrane damage, activation of ion channels, and reactive oxygen species (ROS), all of which promote NLRP3 oligomerization which, along with adaptor protein and caspase-1, forms the inflammasome and cleaves pro-IL-1 $\beta$ and pro-IL-18 into the active IL-1 $\beta$ and IL18 , respectively. ${ }^{49,52,53}$ The last step of this process is the caspase-1-mediated cleavage of Gasdermin D (GSDMD), resulting in the formation of pores in the cell membrane to allow for the release of IL-1 $\beta$ and IL-18 as well as inducing cell death by pyroptosis, producing DAMPs. ${ }^{54,55}$ IL-1 $\beta$ can also further activate the inflammasome (i.e., a feedforward mechanism). ${ }^{3}$ This process collectively intensifies the immune response but, if not tightly regulated, can lead to massive infiltration of activated neutrophils with subsequent NETosis, activation of macrophages, and an exaggerated cytokine response leading to substantial tissue damage. ${ }^{51,56,57}$

In the context of coronaviruses, SARS-CoV directly activates the NLRP3 inflammasomes via its E protein and viroporin 3a. Since significant homology exists between SARS$\mathrm{CoV}$ and SARS-CoV-2, there is likely significant overlap regarding pathophysiology. Both E protein and 3a protein behave as ion channels (viroporins): E protein associates with the ER membrane, causing leakage of $\mathrm{Ca}^{2+}$ into the cytosol; in contrast, viroporin 3a inserts at the cell membrane, promoting $\mathrm{K}^{+}$efflux. ${ }^{54,58}$ The consequent ionic imbalance, activation of various intracellular enzymes, and generation of ROS propagate mitochondrial and lysosomal membrane injury, which activates the NLRP3 inflammasome. Additionally, both E-protein and 3a proteins are found to activate NF-KB independently to drive the transcription of pro-IL-1 $\beta$, proIL-18, and NLRP3. ${ }^{52,53}$ Accordingly, the NLRP3 inflammasome has gained much attention as a potential target for therapy given its central role in the pathogenesis of severe COVID infection.

The liver plays an important role in immune defense, continuously being exposed to pathogens entering the body via the gut and containing the largest collection of fixed macrophages, called Kupffer cells, in the body. ${ }^{59}$ Although Kupffer cells do not normally express ACE2 and, therefore, are not likely the target of direct SARS-2-CoV infection, monocyte-derived macrophages that replenish Kupffer cells in the setting of inflammation may provide a route of extension of the inflammatory stimulus. ${ }^{38}$ Additionally, endothelial cells appear to play an important role. Alveolar endothelial cells are activated in the setting of inflammation, precipitating a hypercoagulable state and neutrophil activation with subsequent NETosis. ${ }^{6}$ Therefore, widespread pyroptosis of macrophages not only in the lung but also in the liver followed by vigorous inflammation and hypercoagulability via endothelial cell activation is perhaps partly responsible for the lobular and portal inflammation, microvascular thrombosis, hepatic sinusoidal congestion, and necrosis observed in liver autopsies of deceased COVID-19 patients (readers are referred to ${ }^{17}$ for more detailed descriptions). This could manifest clinically as hepatomegaly and elevated LFTs, with concomitant jaundice and hepatic encephalopathy, and elevated LDH. Serum LDH, in particular, is used as a marker of various inflammatory states and is significantly elevated in severe COVID-19 patients compared with those without the severe disease and, thus, can be used as a marker for severity and prognosis. ${ }^{60-62}$

\section{Ischemic-Reperfusion Injury}

The liver is highly vascular, and therefore, susceptible to hypoperfusion secondary to circulatory disturbances. Cardiac failure, respiratory failure, and circulatory shock are well established as mechanisms causing passive congestion and hypoperfusion of the liver resulting in centrilobular ischemic necrosis. ${ }^{17,63,64}$ In the context of COVID-19, acute respiratory distress syndrome (ARDS) induces severe hypoxia that leads to ischemic liver damage characterized by steatosis and subsequent hepatocyte death. ${ }^{31}$ This is followed by reperfusion characterized by neutrophil infiltration with the generation of ROS, all of which propagate further liver injury via lipid peroxidation and oxidation of DNA and proteins. ${ }^{10,31}$

Hypoxia independently imposes oxidative stress that promotes the generation of ROS, which have been identified as one of the key triggers of the inflammasome, evidenced by the chemical inhibition of ROS generation curbing inflammasome activation in response to several different stimuli. ${ }^{65}$ The inflammasome-related cytokines IL-1 $\beta$ and IL- 18 as well as DAMPs released by inflammasome-induced pyroptosis will exacerbate reperfusion injury, setting up a self-reinforcing loop of inflammation and tissue damage, and, as such, inflammasome-mediated inflammation is considered a potential therapeutic target to alleviate hepatic $\mathrm{I} / \mathrm{R}$ injury in various surgical settings. ${ }^{66}$ Such principles could potentially translate over to I/R injury in severe COVID-19 cases, where inflammasome inhibition can alleviate symptoms of hypoxic damage. Indeed, I/R resulting in hypoxic hepatitis secondary to anoxia induced by respiratory failure is seen in severe cases. $^{67}$

\section{The Potential Role of Nets in COVID/Liver Disease}

In short, COVID-induced hepatic disease is likely to involve a combination of several mechanisms, including direct infection, cytokine storm and SIRS, thrombotic microangiopathy, and, in severe cases, hypoxic hepatitis. With increasing grades of disease severity, more of these pathomechanisms may get involved to cause liver injury, which manifests clinically as a mild-to-moderate rise of AST/ALT and a concomitant drop in serum albumin in severe cases. ${ }^{7,63}$ The key players mediating each of these processes remain unelucidated, but the usual mediators, endothelial cell damage with neutrophils and platelets, likely play a role. ${ }^{68}$ 
Identifying the major contributors to the pathogenesis of COVID-induced liver disease will likely have significant therapeutic implications, not only by revealing novel therapeutic targets but also perhaps by susceptible patient demographics and reliable prognostic biomarkers.

Recently, NETs have been implicated in the pathogenesis of severe COVID, characterized by a sudden, rapid deterioration of patients culminating as ARDS. ${ }^{15,57,68}$ This hypothesis was supported by neutrophilia, elevated NET markers such as cell-free DNA and histones, and SARS-CoV-2 patient sera being able to induce NET formation. ${ }^{69-71}$ Lastly, the degree of NET marker elevation correlates with pneumonia-associated lung injury. ${ }^{72}$ The role of NETs in the pathophysiology of COVID is thought to be a primary result of vascular damage and the consequent exacerbation of inflammation with hypercoagulability, manifesting clinically as ARDS and multi-organ failure, including the liver. ${ }^{21,73,74}$ Indeed, liver injury is a consistent feature of COVID-19, with histopathological evidence demonstrating patchy necrotic areas akin to NET-damaged livers. ${ }^{75}$

The liver functions as a frontline immune organ due to its unique blood supply, filtering the blood of any pathogens or PAMPs entering via the gut and subsequently eliciting appropriate immune responses. Furthermore, the liver has been reported to be the primary organ for bacterial sequestration which is thought to be fundamentally through the actions of two immune cells: Kupffer cells, the intrasinusoidal resting macrophages of the liver, through phagocytosis, and neutrophils, which are recruited to the liver in the setting of infection, via NET formation. ${ }^{71,76}$ Indeed, apart from COVID, NETs have been well-established as key players in propagating liver injury in several pathologies, including alcohol-associated liver injury, portal hypertension in chronic liver disease, sepsis, liver transplantation, and even cancer. ${ }^{10,18}$

Potential Role of NETs in COVID-Induced Endotheliopathy The role of NETs in multiorgan failure characterizing severe COVID-19 has been confirmed in several studies. ${ }^{13,21,51,75}$ Both arterial and venous thrombosis are detected in COVID19 patients, causing micro- and macrovascular thrombotic phenomena including acute coronary syndrome, deep vein thrombosis, and pulmonary embolism, with concomitant elevation in NET markers as well as neutrophil-platelet aggregates. ${ }^{69,77}$ However, a current limitation in the study of NETs in COVID-19 is the lack of data on their role in mildto-moderate COVID infections. COVID-19 patients, regardless of disease severity, demonstrate neutrophilia comprising of immature neutrophils, which are known to show increased NETosis at baseline. ${ }^{68,75,78}$ As such, the role of NETs in mild-to-moderate disease may also exist. In the context of liver disease, elevated AST/ALT levels are also seen in non-severe COVID-19 patients. ${ }^{9}$ With the current opinion that multiorgan manifestations of COVID-19 are secondary to a hyperinflammatory cytokine storm, why do moderate cases, which do not exhibit a hyperinflammatory state, exhibit elevated LFTs?

Intriguingly, several recent studies have suggested that accepting a cytokine storm as the principal mediator of
COVID-19 may be premature. These postulations are based on the magnitude of serum cytokine elevations being insufficient to cause such symptoms. ${ }^{79,80}$ To this end, a recent review article stated that organ-specific cytokines should be investigated. ${ }^{68}$ In such a scenario, NETs may also be involved. Mounting evidence suggests that the pathophysiology of COVID-19 revolves around endothelial cell damage-so-called endotheliitis or endotheliopathy. ${ }^{81-84}$ ACE2 is expressed on endothelial cells, and SARS-CoV-2 has consistently been detected within endothelial cells. ${ }^{63,81,85}$ Endothelial cell activation and damage expose subendothelial collagen and other thrombogenic substances which attract platelets and neutrophils to collectively promote NETosis. ${ }^{24}$ NETs can also directly activate endothelial cells as well as induce endothelial cell death through histones. ${ }^{23,86,87}$ Therefore, since immature neutrophilia and elevated NET markers are observed in COVID-19 patients, their potential accumulation in the liver may represent a key trigger for endothelial cell injury and microvascular thrombotic events in COVID-19 patients in severe as well as non-severe cases.

\section{Sepsis}

Sepsis is characterized by a deadly inflammatory syndrome secondary to a dysfunctional immune response to infection. The liver is principally responsible for the clearance of bacteremia in the setting of sepsis through Kupffer cells by phagocytosis and neutrophils through NET formation. Liver sinusoidal endothelial cells (LSECs) upregulate TLR4 on their surface secondary to infection, facilitating retention of neutrophils within hepatic sinusoids by mediating neutrophil adherence to hyaluronan via CD44. ${ }^{10,88}$ Hypoxia-induced injury of LSECs promotes the surface expression of p-selectin and von-Willebrand factor, which contributes to the recruitment and activation of neutrophils. The result of this is two-fold: NET production in the liver exceeds that of other tissues and, due to increased retention of NETs, the liver may also retain circulating NETs originally produced in other organs. ${ }^{82,88}$

While NET formation contributes significantly to bacterial sequestration and, therefore, plays a key role in immune defense, the high intrahepatic concentration of NETs may not only contribute to enhanced microbial sequestration but also, through its cytotoxic elements including but not limited to histones, $\mathrm{NE}$, and cathepsin $\mathrm{G}$, damage the liver and induce or exacerbate inflammatory responses and propagate further liver injury. Accompanying the inflammatory response in sepsis is disseminated intravascular coagulation (DIC), which is characterized by a consumption coagulopathy. NETs are thought to contribute to DIC through binding platelet, RBCs, and fibrinogen to precipitate thrombus formation. $24,57,89$ Negatively charged DNA activates the intrinsic pathway of the coagulation cascade, and histones promote a hypercoagulable state by causing endothelial cell damage. Proteases within NETs, such as NE, are thought to activate the intrinsic and extrinsic pathways of coagulation. ${ }^{90}$ Accordingly, research studying the significance of NET-mediated liver damage reports that the genetic inhibition of NET formation and various components of NETs greatly ameliorate symptoms of liver damage. ${ }^{10,91}$ 
The endogenous stimulants of NET formation are believed to be histones, hypoxia, and high mobility group box protein 1 (HMGB1), which are released by irreversibly injured cells and function as DAMPs. ${ }^{86,92,93}$ A study showed that treating neutrophils with exogenous histones or HMGB1 demonstrated a proportional increase in NET formation. ${ }^{20}$ HMGB1 is a nonhistone protein normally linked to chromatin. Its translocation to the cytoplasm and subsequent extrusion into the extracellular space are mediated by high levels of NLRP3 inflammasome activity in immune cells, which simultaneously cause pyroptosis resulting in the production of more DAMPs to augment the inflammatory response. ${ }^{54}$ Given the exaggerated NLRP3 inflammasome activity characteristic of severe COVID infection and its role in propagating the cytokine storm, inflammasome-mediated HMGB1 release into the extracellular space may induce excessive NET formation which further exacerbates the disease.

\section{Conclusion and Perspectives}

Collectively, we propose that NETs-observed to be elevated in COVID infection-play principal roles in liver injury in addition to acute lung injury. In support of our findings, serum NET markers are elevated in COVID-19 patients, which correlate with disease severity ${ }^{70,71}$; well-established inducers of NETosis, such as endothelial cells and proinflammatory cytokine IL-1 $\beta$, are confirmed to play a major role in COVID-19 pathogenesis, ${ }^{51,55,94}$ NETs play key roles in liver disease regardless of etiology, ${ }^{10}$ and other, similar, immunerelated diseases feature NETs, ${ }^{12,26,95}$ and NETs are consistently detected within pulmonary, ${ }^{75,96}$ renal $^{16}{ }^{16}$ and cardiac ${ }^{97}$ microthrombi in COVID-19 patients.

Notably, the prognostic significance of LFT abnormalities in COVID-19 patients remains uncertain. However, since the onset of the pandemic, concerns have been raised about the susceptibility of chronic liver disease patients of COVID-19, 7,30 as these conditions share many of the same risk factors, including age, obesity, and diabetes. Additionally, advancing liver disease is associated with metabolic/endocrine derangements, immune dysregulation, and coagulopathy, all of which increase the risk of severe COVID-19. ${ }^{30}$ Therefore, future work should aim to substantiate the prognostic value of LFTs in COVID-19 patients and, in keeping with the present discussion, evaluate potential relationships between liver abnormalities and NETs to inform therapeutic strategies to ameliorate this condition.

Further research is required to confirm the role of NETs in COVID-19. This holds potential therapeutic significance, as inhibition of NETosis could alleviate symptoms of ARDS and liver injury, as well as other organs because ARDS creates a hypoxic internal environment and liver injury manifests as metabolic disturbances detrimental to all organ systems. A likely challenge in the clinical application of such drugs will be the identification of appropriate patient demographics in which these drugs are indicated. Indeed, the potential clinical benefit of NET inhibition has to be counterbalanced with the possible deleterious effects these inhibiting key immune mediators could have on the patient trajectory. Nevertheless, given the central role of NETs in mediating lung, liver, kidney, and cardiac pathologies in COVID-19, numerous clinical trials are ongoing to evaluate the efficacy DNase-1 (dornase-alfa), a NET-lysing drug, in combating COVID-19. ${ }^{98}$

\section{Funding \\ None.}

Conflict of Interest

None declared.

\section{References}

1 Yaqinuddin A, Kashir J. Innate immunity in COVID-19 patients mediated by NKG2A receptors, and potential treatment using Monalizumab, Cholroquine, and antiviral agents. Med Hypotheses 2020;140:109777

2 Giamarellos-Bourboulis EJ, Netea MG, Rovina N, et al. Complex immune dysregulation in COVID-19 patients with severe respiratory failure. Cell Host Microbe 2020;27(06):992-1000.e3

3 Yaqinuddin A, Kashir J. Novel therapeutic targets for SARS-CoV-2induced acute lung injury: targeting a potential IL-1 $\beta /$ neutrophil extracellular traps feedback loop. Med Hypotheses 2020; 143:109906

4 Kashir J, Yaqinuddin A. Loop mediated isothermal amplification (LAMP) assays as a rapid diagnostic for COVID-19. Med Hypotheses 2020;141:109786

5 Wang D, Hu B, Hu C, et al. Clinical characteristics of 138 hospitalized patients with 2019 novel coronavirus-infected pneumonia in Wuhan, China. JAMA 2020;323(11):1061-1069

6 Luglio M, Tannuri U, de Carvalho WB, et al. COVID-19 and liver damage: narrative review and proposed clinical protocol for critically ill pediatric patients. Clinics (São Paulo) 2020;75:e2250

7 Zhang C, Shi L, Wang FS. Liver injury in COVID-19: management and challenges. Lancet Gastroenterol Hepatol 2020;5(05):428-430

8 Lagana SM, Kudose S, Iuga AC, et al. Hepatic pathology in patients dying of COVID-19: a series of 40 cases including clinical, histologic, and virologic data. Mod Pathol 2020;33(11):2147-2155

9 Amin M. COVID-19 and the liver: overview. Eur J Gastroenterol Hepatol 2021;33(03):309-311

10 Hilscher MB, Shah VH. Neutrophil extracellular traps and liver disease. Semin Liver Dis 2020;40(02):171-179

11 Nirmala JG, Lopus M. Cell death mechanisms in eukaryotes. Cell Biol Toxicol 2020;36(02):145-164

12 Papayannopoulos V. Neutrophil extracellular traps in immunity and disease. Nat Rev Immunol 2018;18(02):134-147

13 Kvietys PR, Fakhoury HMA, Kadan S, Yaqinuddin A, Al-Mutairy E, Al-Kattan K. COVID-19: lung-centric immunothrombosis. Front Cell Infect Microbiol 2021;11:679878

14 Yaqinuddin A, Kvietys P, Kashir J. COVID-19: role of neutrophil extracellular traps in acute lung injury. Respir Investig 2020;58 (05):419-420

15 Wang J, Li Q Yin Y, et al. Excessive neutrophils and neutrophil extracellular traps in COVID-19. Front Immunol 2020;11:2063

16 Schurink B, Roos E, Radonic T, et al. Viral presence and immunopathology in patients with lethal COVID-19: a prospective autopsy cohort study. Lancet Microbe 2020;1(07):e290-e299

17 Caramaschi S, Kapp ME, Miller SE, et al. Histopathological findings and clinicopathologic correlation in COVID-19: a systematic review. Mod Pathol 2021;34(09):1614-1633

18 Klopf J, Brostjan C, Eilenberg W, Neumayer C. Neutrophil extracellular traps and their implications in cardiovascular and inflammatory disease. Int J Mol Sci 2021;22(02):559

19 Zhong P, Xu J, Yang D, et al. COVID-19-associated gastrointestinal and liver injury: clinical features and potential mechanisms. Signal Transduct Target Ther 2020;5(01):256 
20 Huang $\mathrm{H}$, Tohme S, Al-Khafaji AB, et al. Damage-associated molecular pattern-activated neutrophil extracellular trap exacerbates sterile inflammatory liver injury. Hepatology 2015;62 (02):600-614

21 Janiuk K, Jabłońska E, Garley M. Significance of NETs Formation in COVID-19. Cells 2021;10(01):151

22 Iliadi V, Konstantinidou I, Aftzoglou K, Iliadis S, Konstantinidis TG, Tsigalou $\mathrm{C}$. The emerging role of neutrophils in the pathogenesis of thrombosis in covid-19. Int J Mol Sci 2021;22(10):5368

23 Gould TJ, Lysov Z, Liaw PC. Extracellular DNA and histones: double-edged swords in immunothrombosis. J Thromb Haemost 2015;13(Suppl 1):S82-S91

24 Thålin C, Hisada Y, Lundström S, Mackman N, Wallén H. Neutrophil extracellular traps: villains and targets in arterial, venous, and cancer-associated thrombosis. Arterioscler Thromb Vasc Biol 2019;39(09):1724-1738

25 Laridan E, Martinod K, De Meyer SF. Neutrophil extracellular traps in arterial and venous thrombosis. Semin Thromb Hemost 2019; 45(01):86-93

26 Bonaventura A, Vecchié A, Abbate A, Montecucco F. Neutrophil extracellular traps and cardiovascular diseases: an update. Cells 2020;9(01):E231

27 Huang C, Wang Y, Li X, et al. Clinical features of patients infected with 2019 novel coronavirus in Wuhan, China. Lancet 2020;395 (10223):497-506

28 Zhou F, Yu T, Du R, et al. Clinical course and risk factors for mortality of adult inpatients with COVID-19 in Wuhan, China: a retrospective cohort study. Lancet 2020;395(10229):1054-1062

29 Xu Z, Shi L, Wang Y, et al. Pathological findings of COVID-19 associated with acute respiratory distress syndrome. Lancet Respir Med 2020;8(04):420-422

30 Marjot T, Webb GJ, Barritt AS IV, et al. COVID-19 and liver disease: mechanistic and clinical perspectives. Nat Rev Gastroenterol Hepatol 2021;18(05):348-364

31 Tian D, Ye Q. Hepatic complications of COVID-19 and its treatment. J Med Virol 2020;92(10):1818-1824

32 Hoffmann M, Kleine-Weber H, Schroeder S, et al. SARS-CoV-2 cell entry depends on ACE2 and TMPRSS2 and is blocked by a clinically proven protease inhibitor. Cell 2020;181(02):271-280.e8

33 Shukla A, Mohanka R. COVID 19 and the liver. J Assoc Physicians India 2020;68(11):11-12

34 Ali N. Relationship between COVID-19 infection and liver injury: a review of recent data. Front Med (Lausanne) 2020;7:458

35 Krishna M. Patterns of necrosis in liver disease. Clin Liver Dis (Hoboken) 2017;10(02):53-56

36 Mushtaq K, Khan MU, Iqbal F, et al. NAFLD is a predictor of liver injury in COVID-19 hospitalized patients but not of mortality, disease severity on the presentation or progression - The debate continues. J Hepatol 2021;74(02):482-484

37 Ji D, Qin E, Xu J, et al. Non-alcoholic fatty liver diseases in patients with COVID-19: A retrospective study. J Hepatol 2020;73(02): 451-453

38 Nardo AD, Schneeweiss-Gleixner M, Bakail M, Dixon ED, Lax SF, Trauner M. Pathophysiological mechanisms of liver injury in COVID-19. Liver Int 2021;41(01):20-32

39 Wang Y, Liu S, Liu H, et al. SARS-CoV-2 infection of the liver directly contributes to hepatic impairment in patients with COVID-19. J Hepatol 2020;73(04):807-816

40 Hay N, Sonenberg N. Upstream and downstream of mTOR. Genes Dev 2004;18(16):1926-1945

41 Hsu PP, Kang SA, Rameseder J, et al. The mTOR-regulated phosphoproteome reveals a mechanism of mTORC1-mediated inhibition of growth factor signaling. Science 2011;332(6035):1317-1322

42 Porstmann T, Santos CR, Griffiths B, et al. SREBP activity is regulated by mTORC1 and contributes to Akt-dependent cell growth. Cell Metab 2008;8(03):224-236

43 Kindrachuk J, Ork B, Hart BJ, et al. Antiviral potential of ERK/MAPK and PI3K/AKT/mTOR signaling modulation for Middle East respi- ratory syndrome coronavirus infection as identified by temporal kinome analysis. Antimicrob Agents Chemother 2015;59(02): 1088-1099

44 Cottam EM, Maier HJ, Manifava M, et al. Coronavirus nsp6 proteins generate autophagosomes from the endoplasmic reticulum via an omegasome intermediate. Autophagy 2011;7(11): 1335-1347

45 Bolourian A, Mojtahedi Z. Obesity and COVID-19: the mTOR pathway as a possible culprit. Obes Rev 2020;21(09):e13084

46 Lee C, Choi WJ. Overview of COVID-19 inflammatory pathogenesis from the therapeutic perspective. Arch Pharm Res 2021;44 (01):99-116

$47 \mathrm{Kim}$ JS, Lee JY, Yang JW, et al. Immunopathogenesis and treatment of cytokine storm in COVID-19. Theranostics 2021;11(01): 316-329

48 Lucas C, Wong P, Klein J, et al; Yale IMPACT Team. Longitudinal analyses reveal immunological misfiring in severe COVID-19. Nature 2020;584(7821):463-469

49 Kelley N, Jeltema D, Duan Y, He Y. The NLRP3 inflammasome: an overview of mechanisms of activation and regulation. Int J Mol Sci 2019;20(13):3328

50 López-Reyes A, Martinez-Armenta C, Espinosa-Velázquez R, et al. NLRP3 inflammasome: the stormy link between obesity and COVID-19. Front Immunol 2020;11:570251

51 Kashir J, Ambia AR, Shafqat A, Sajid MR, AlKattan K, Yaqinuddin A. Scientific premise for the involvement of neutrophil extracellular traps (NETs) in vaccine-induced thrombotic thrombocytopenia (VITT). Leukocyte Biol 2021 JLB.5COVR0621-320RR. Doi: 10.1002/ JLB.5COVR0621-320RR

52 Yap JKY, Moriyama M, Iwasaki A. Inflammasomes and pyroptosis as therapeutic targets for COVID-19. J Immunol 2020;205(02): 307-312

53 Jo E-KK, Kim JK, Shin D-MM, Sasakawa C. Molecular mechanisms regulating NLRP3 inflammasome activation. Cell Mol Immunol 2016;13(02):148-159

54 van den Berg DF, Te Velde AA. Severe COVID-19: NLRP3 inflammasome dysregulated. Front Immunol 2020;11:1580

55 Rodrigues TS, de Sá KSG, Ishimoto AY, et al. Inflammasomes are activated in response to SARS-CoV-2 infection and are associated with COVID-19 severity in patients. J Exp Med 2021;218(03): e20201707

56 Tomar B, Anders H-JJ, Desai J, Mulay SR. Neutrophils and neutrophil extracellular traps drive necroinflammation in COVID-19. Cells 2020;9(06):1383

57 Denning NL, Aziz M, Gurien SD, Wang P. DAMPs and NETs in sepsis. Front Immunol 2019;10(Oct):2536

58 Chen I-YY, Moriyama M, Chang M-FF, Ichinohe T. Severe acute respiratory syndrome coronavirus viroporin 3a activates the NLRP3 inflammasome. Front Microbiol 2019;10(Jan):50

59 Kubes P, Jenne C. Immune responses in the liver. Annu Rev Immunol 2018;36(01):247-277

60 Henry BM, Aggarwal G, Wong J, et al. Lactate dehydrogenase levels predict coronavirus disease 2019 (COVID-19) severity and mortality: a pooled analysis. Am J Emerg Med 2020;38(09): 1722-1726

61 Henry BM, de Oliveira MHS, Benoit S, Plebani M, Lippi G. Hematologic, biochemical and immune biomarker abnormalities associated with severe illness and mortality in coronavirus disease 2019 (COVID-19): a meta-analysis. Clin Chem Lab Med 2020;58 (07):1021-1028

62 Szarpak L, Ruetzler K, Safiejko K, et al. Lactate dehydrogenase level as a COVID-19 severity marker. Am J Emerg Med 2021; 45:638-639

63 Gupta A, Madhavan MV, Sehgal K, et al. Extrapulmonary manifestations of COVID-19. Nat Med 2020;26(07):1017-1032

64 Vasquez-Bonilla WO, Orozco R, Argueta V, et al. A review of the main histopathological findings in coronavirus disease 2019. Hum Pathol 2020;105:74-83 
65 Yang Y, Wang H, Kouadir M, Song H, Shi F. Recent advances in the mechanisms of NLRP3 inflammasome activation and its inhibitors. Cell Death Dis 2019;10(02):128

66 Jiménez-Castro MB, Cornide-Petronio ME, Gracia-Sancho J, Peralta C. Inflammasome-mediated inflammation in liver ischemiareperfusion injury. Cells 2019;8(10):E1131

67 Sun J, Aghemo A, Forner A, Valenti L. COVID-19 and liver disease. Liver Int 2020;40(06):1278-1281

68 Osuchowski MF, Winkler MS, Skirecki T, et al. The COVID-19 puzzle: deciphering pathophysiology and phenotypes of a new disease entity. Lancet Respir Med 2021;9(06):622-642

69 Middleton EA, He X-Y, Denorme F, et al. Neutrophil extracellular traps contribute to immunothrombosis in COVID-19 acute respiratory distress syndrome. Blood 2020;136(10):1169-1179

70 Ondracek AS, Lang IM. Neutrophil extracellular traps as prognostic markers in COVID-19: a welcome piece to the puzzle. Arterioscler Thromb Vasc Biol 2021;41(02):995-998

$71 \mathrm{Ng} \mathrm{H}$, Havervall S, Rosell A, et al. Circulating markers of neutrophil extracellular traps are of prognostic value in patients with COVID19. Arterioscler Thromb Vasc Biol 2021;41(02):988-994

72 Radermecker C, Detrembleur N, Guiot J, et al. Neutrophil extracellular traps infiltrate the lung airway, interstitial, and vascular compartments in severe COVID-19. J Exp Med 2020;217(12):e20201012

73 Veras FP, Pontelli MC, Silva CM, et al. SARS-CoV-2-triggered neutrophil extracellular traps mediate COVID-19 pathology. J Exp Med 2020;217(12):e20201129

74 Arcanjo A, Logullo J, Menezes CCB, et al. The emerging role of neutrophil extracellular traps in severe acute respiratory syndrome coronavirus 2 (COVID-19). Sci Rep 2020;10(01):19630

75 Ackermann M, Anders H-J, Bilyy R, et al. Patients with COVID-19: in the dark-NETs of neutrophils. Cell Death Differ 2021;28(11): 3125-3139

76 Meijenfeldt FAV, Jenne CN. Netting liver disease: neutrophil extracellular traps in the initiation and exacerbation of liver pathology. Semin Thromb Hemost 2020;46(06):724-734

77 Petito E, Falcinelli E, Paliani U, et al; COVIR study investigators. Association of neutrophil activation, more than platelet activation, with thrombotic complications in coronavirus disease 2019. $\mathrm{J}$ Infect Dis 2021;223(06):933-944

78 Hazeldine J, Lord JM. Neutrophils and COVID-19: active participants and rational therapeutic targets. Front Immunol 2021; 12:680134

79 Sinha P, Matthay MA, Calfee CS. Is a "cytokine storm" relevant to COVID-19? JAMA Intern Med 2020;180(09):1152-1154

80 Kox M, Waalders NJB, Kooistra EJ, Gerretsen J, Pickkers P. Cytokine levels in critically ill patients with COVID-19 and other conditions. JAMA 2020;324(15):1565-1567

81 Nascimento Conde J, Schutt WR, Gorbunova EE, Mackow ER. Recombinant ACE2 expression is required for SARS-CoV-2 to infect primary human endothelial cells and induce inflammatory and procoagulative responses. MBio 2020;11(06):1-7

82 Cugno M, Meroni PL, Gualtierotti R, et al. Complement activation and endothelial perturbation parallel COVID-19 severity and activity. J Autoimmun 2021;116:102560
83 Perico L, Benigni A, Casiraghi F, Ng LFP, Renia L, Remuzzi G. Immunity, endothelial injury and complement-induced coagulopathy in COVID-19. Nat Rev Nephrol 2021;17(01): 46-64

84 Yaqinuddin A, Kashir J. The central role of neutrophil extracellular traps in SARS-CoV-2-induced thrombogenesis and vasculitis. African J Respir Med. 2020;15(02)

85 Nuovo GJ, Magro C, Shaffer T, et al. Endothelial cell damage is the central part of COVID-19 and a mouse model induced by injection of the S1 subunit of the spike protein. Ann Diagn Pathol 2021; $51: 151682$

86 Saffarzadeh M, Juenemann C, Queisser MA, et al. Neutrophil extracellular traps directly induce epithelial and endothelial cell death: a predominant role of histones. PLoS One 2012;7 (02):e32366

$87 \mathrm{Xu}$ J, Zhang X, Pelayo R, et al. Extracellular histones are major mediators of death in sepsis. Nat Med 2009;15(11):1318-1321

88 Honda M, Kubes P. Neutrophils and neutrophil extracellular traps in the liver and gastrointestinal system. Nat Rev Gastroenterol Hepatol 2018;15(04):206-221

89 Gollomp K, Kim M, Johnston I, et al. Neutrophil accumulation and NET release contribute to thrombosis in HIT. JCI Insight 2018;3 (18):1-14

90 Massberg S, Grahl L, von Bruehl ML, et al. Reciprocal coupling of coagulation and innate immunity via neutrophil serine proteases. Nat Med 2010;16(08):887-896

91 Kolaczkowska E, Jenne CN, Surewaard BGJ, et al. Molecular mechanisms of NET formation and degradation revealed by intravital imaging in the liver vasculature. Nat Commun 2015; 6:6673

92 Street ME. HMGB1: a possible crucial therapeutic target for COVID-19? Horm Res Paediatr 2020;93(02):73-75

93 Maugeri N, Campana L, Gavina M, et al. Activated platelets present high mobility group box 1 to neutrophils, inducing autophagy and promoting the extrusion of neutrophil extracellular traps. J Thromb Haemost 2014;12(12):2074-2088

94 Saeedi-Boroujeni A, Mahmoudian-Sani MR, Nashibi R, Houshmandfar S, Tahmaseby Gandomkari S, Khodadadi A. Tranilast: a potential anti-Inflammatory and NLRP3 inflammasome inhibitor drug for COVID-19. Immunopharmacol Immunotoxicol 2021;43 (03):247-258

95 Söderberg D, Segelmark M. Neutrophil extracellular traps in ANCA-associated vasculitis. Front Immunol 2016;7(Jun):256

96 Ackermann M, Verleden SE, Kuehnel M, et al. Pulmonary vascular endothelialitis, thrombosis, and angiogenesis in Covid-19. N Engl J Med 2020;383(02):120-128

97 Blasco A, Coronado M-J, Hernández-Terciado F, et al. Assessment of neutrophil extracellular traps in coronary thrombus of a case series of patients with COVID-19 and myocardial infarction. JAMA Cardiol 2020;6(04):469-474

98 DISmantling COvid iNduced Neutrophil ExtraCellular Traps (DISCONNECT-1) - Full Text View - ClinicalTrials.gov. Accessed September 15, 2021 at: https://clinicaltrials.gov/ct2/show/NCT04409925?term=Neutrophil+extracellular+trap\&cond=COVID-19\&draw=2\&rank=2 